\title{
Estudo de seguimento de utentes após realização de um eletrocardiograma em Medicina Geral e Familiar
}

Paulo Santos, ${ }^{*}$ Alberto Hespanhol,* Luciana Couto**

\section{RESUMO}

Introdução: O eletrocardiograma (ECG) é um exame do quotidiano em Medicina Geral e Familiar (MGF), usado no estudo e seguimento de doenças relacionadas com o coração.

Objetivo: Caracterizar a alteração clínica a médio prazo no seguimento dos utentes a quem foi realizado um ECG na consulta de MGF.

Métodos: Estudo observacional longitudinal prospetivo dos ECG requisitados e realizados no Centro de Saúde de S. João, Porto, desde $01 / 03 / 2007$ até $28 / 02 / 2009$. Na requisição foi preenchido um protocolo que incluía o motivo para o pedido, a sintomatologia dos doentes, os seus antecedentes clínicos, e registado o resultado da leitura. Seis meses após, os processos clínicos foram avaliados para verificar a alteração à orientação clínica previamente definida para o utente. Usou-se um modelo de regressão logística multinominal para descrever a associação entre a alteração ao seguimento dos doentes e o motivo para a requisição dos exames e o seu resultado, classificado segundo o Novacode.

Resultados: Foram avaliados 870 ECG de 817 utentes, 56,4\% do sexo feminino, com uma mediana de idades de 57 anos. $O$ principal motivo para a requisição dos ECG foi a presença de sintomatologia na consulta (48,5\%). 54,5\% foram normais e $9,7 \%$ apresentaram alterações major. Em 67,9\% (IC95\%:64,8-70,0\%) mantiveram-se os cuidados previamente instituídos. A taxa de referenciação aos cuidados hospitalares foi de 5,7\% (IC95\%:4,8-7,5\%). Na análise multivariada, a decisão na orientação dos utentes foi significativamente influenciada pelos resultados dos ECG, nos utentes em que o ECG é requisitado para seguimento de doença previamente diagnosticada mas não nos que apresentam sintomas na consulta.

Conclusão: A maioria dos utentes que realizam um ECG na consulta de MGF mantém inalterados os cuidados instituídos previamente à requisição do exame, verificando-se uma maior utilidade nos casos de seguimento de fatores de risco cardiovasculares e de doença cardíaca prévia perante a existência de alterações eletrocardiográficas.

Palavras-chave: Eletrocardiografia; Medicina Geral e Familiar; Gestão da Doença; Anamnese.

\section{INTRODUÇÃO}

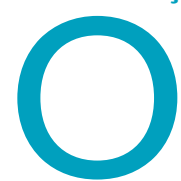

eletrocardiograma (ECG), enquanto técnica de registo da corrente elétrica cardíaca, foi descrito para utilização humana em 1902 no historicamente célebre artigo "Galvanometrische registratie van het menschilijk electrocardiogram" ${ }^{1}$ por Willem Einthoven, tendo utilização na investigação e seguimento das doenças relacionadas com o coração.

Trata-se de um exame quotidiano em Medicina Geral e

* USF de S. João do Porto, Departamento de Clínica Geral/FMUP

** Unidade de Saúde Familiar das Camélias, Departamento de Clínica Geral/FMUP
Familiar $^{2,3} \mathrm{e}$, apesar da profícua idade, mantém e renova a sua utilidade na prática clínica diária, ${ }^{4}$ sendo reconhecida a existência de diferenças entre a prática dos Cuidados Primários e Hospitalares. ${ }^{5,6}$

Em Portugal foram realizados, ao nível dos Cuidados de Saúde Primários, em 2007, mais de um milhão de ECG, representando em média $14,8 \%$ da população utilizadora dos Centros de Saúde e 3,9 \% das consultas médicas efetuadas. ${ }^{7}$ Em relação a 1997, onde o ECG foi realizado em $3,2 \%$ das consultas, ${ }^{8}$ representa um acréscimo de exames de $9,6 \%$. 
Este volume de requisições encontra paralelismo no que acontece noutros países com valores que oscilam entre os 2,7 e os $4,5 \%$ das consultas médicas, ${ }^{9,10}$ e depende de características intrínsecas ao doente, de características relativas ao prestador de cuidados e de condicionantes do sistema de saúde.

O principal motivo para a sua requisição prende-se com a existência de sintomatologia cardíaca, como dor torácica e palpitações, ou seguimento de doentes com fatores de risco coronário, sobretudo a hipertensão arterial, ${ }^{11}$ aparecendo também o exame global de saúde como relevante, ${ }^{12}$ apesar de a evidência apontar no sentido contrário.

Apesar de basearem a decisão de requisitar o exame sobretudo em motivos de ordem clínica, a perspetiva de alteração do plano de seguimento em função da previsão do resultado do ECG é baixa nos Médicos de Família, onde cerca de metade não previa alterar a orientação à partida, independentemente do resultado do exame. No estudo de Rutten ${ }^{11}$ este valor baixou para $31 \%$ de alterações ao seguimento após conhecer a leitura do ECG.

A prática dos Cuidados de Saúde Primários apresenta variações internacionais fruto da diferente organização dos serviços de saúde e do nível de diferenciação conferido aos respetivos profissionais. Coloca-se então a questão de saber se em Portugal estes dados se confirmam.

O objetivo deste estudo é caracterizar a alteração clínica a médio prazo no seguimento dos utentes a quem foi realizado um ECG no contexto da consulta de Medicina Geral e Familiar.

\section{MÉTODOS}

Realizou-se um estudo observacional longitudinal, onde se estudaram os ECG realizados no Centro de Saúde de S. João, requisitados no contexto da consulta de Medicina Geral e Familiar de forma consecutiva desde 01/03/2007, por um período de 24 meses, por 9 Médicos de Família e realizados nas instalações do Centro de Saúde. Foram excluídos os ECG realizados por entidades externas.

O Centro de Saúde de S. João corresponde à parte assistencial do Projeto “Tubo de Ensaio”, num protocolo estabelecido com a Administração Regional de Saúde do Norte, onde o desenvolvimento de projetos inovadores nas áreas da Administração e da Prestação de Cuidados e a formação pré e pós-graduada em Medicina Geral e Familiar foram complementados pela contratualização da prestação de Cuidados de Saúde Primários em Medicina Geral e
Familiar a uma população de cerca de 20.000 utentes na cidade do Porto. ${ }^{13}$

No início, os ECGs requisitados na consulta dos Médicos de Família foram realizados nas instalações do Centro de Saúde por enfermeiros treinados para o efeito e enviados para relatar a um cardiologista, e desde 2005 passaram a ser avaliados por um grupo de 3 Médicos de Família e relatados diretamente no processo clínico dos utentes.

No momento da requisição de um ECG, o médico preenchia uma folha estruturada de colheita de dados que incluía dados demográficos, o motivo clínico que justificava a requisição, a presença de sintomatologia relacionada com o sistema cardiovascular, a presença de fatores de risco cardiovasculares e a presença de antecedentes cardíacos. Os dados omissos foram completados por pesquisa manual dos processos clínicos.

Procedeu-se à categorização dos motivos para a requisição do exame em (1) presença de sintomatologia na consulta, (2) seguimento de doença cardiovascular previamente diagnosticada, e (3) exame global de saúde.

Os ECG foram realizados por enfermeiros treinados num aparelho Cardiette start $100 \mathrm{H}$ e relatados por médicos de família com experiência na leitura sendo registados no processo clínico eletrónico. Para efeitos de análise procedeuse à codificação pelo Novacode,${ }^{14}$ tendo-se classificado em normal, com anormalidades minor ou com anormalidades major.

Seis meses depois os processos foram analisados de forma manual no sentido de registar as alterações à orientação clínica prévia à realização do ECG, em termos de (1) manutenção dos cuidados anteriormente instituídos, (2) pedido de outros exames, (3) alteração da medicação previamente instituída, (4) referência aos cuidados hospitalares, e (5) morte.

\section{Análise estatística}

Os intervalos de confiança foram calculados pelo método modificado de Wald. A alteração ao seguimento foi categorizada em variáveis dummy, utilizando-se a regressão logística univariada nas covariáveis para a determinação dos valores de odds ratio e respetivos intervalos de confiança.

Um modelo de regressão logística multinominal foi utilizado para descrever a associação entre a alteração ao seguimento dos doentes e o motivo para a requisição dos exames e o seu resultado segundo a classificação de Nova- 


\begin{tabular}{|c|c|c|}
\hline \multicolumn{3}{|c|}{$\begin{array}{l}\text { QUADRO I. Caracterização da população. } \\
\text { (ECG - eletrocardiograma; IC95\% - intervalo de confiança a 95\%). }\end{array}$} \\
\hline ECG (n) & 870 & \\
\hline Utentes ( $n$ ) & 817 & \\
\hline Masculino / Feminino & $356 / 461$ & $43,6 / 56,4 \%$ \\
\hline Idade (mediana; distância interquartil) & 57 & $42-72$ anos \\
\hline Masculino (mediana; distância interquartil) & 53 & $38-69$ anos \\
\hline Feminino (mediana; distância interquartil) & 59 & $44-74$ anos \\
\hline ECG / médico (média; IC95\%) & 96,7 & $50,7-142,6$ \\
\hline Presença de sintomas cardíacos (\%, IC95\%) & 54,8 & $51,5-58,1 \%$ \\
\hline Antecedentes pessoais cardiovasculares (\%, IC95\%) & 15,7 & $13,4-18,3 \%$ \\
\hline Presença de fatores de risco cardiovasculares (\%, IC95\%) & 69,2 & $66,1-72,2 \%$ \\
\hline
\end{tabular}

cuidados previamente instituídos. Tratando-se de variáveis polinominais, utilizou-se como comparador o exame global de saúde nos motivos para a requisição do exame, e o ECG normal nos resultados. A seleção das variáveis de ajustamento teve em conta a significância estatística da análise univariada. Aceitou-se um erro alfa de 0,05.

\section{RESULTADOS}

Foram avaliados 870 ECGs de 817 utentes (56,4\% do sexo feminino) com uma mediana de idades de 57 anos (distância interquartil:

code. Os óbitos foram retirados da análise. Para comparador da variável de resultado utilizou-se a manutenção dos
42-72 anos), sendo de 53 anos para o sexo masculino (distância interquartil: 38-69 anos) e de 59 anos para o sexo fe-

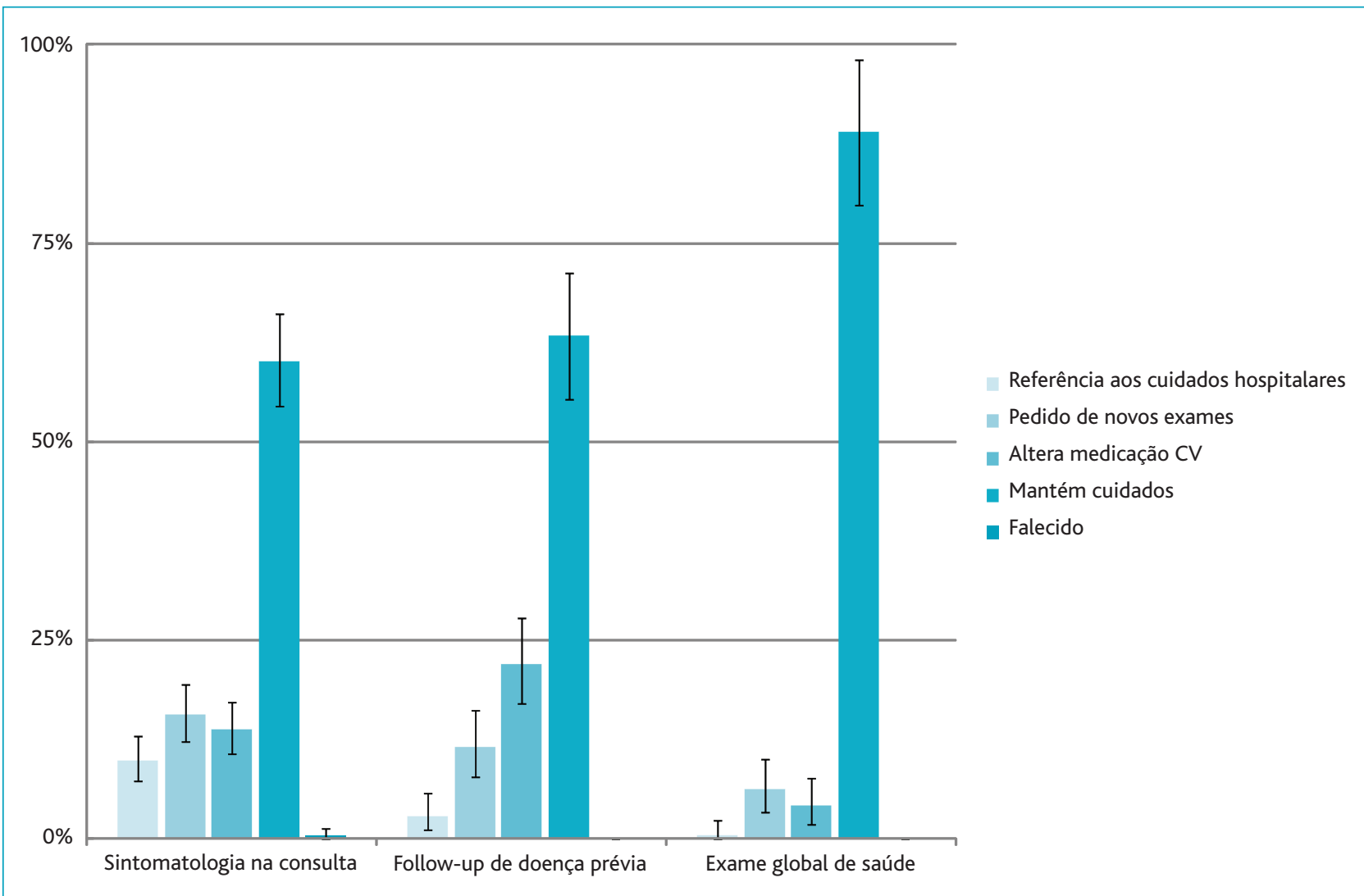

Figura 1. Estudo do seguimento dos utentes em função do motivo para a requisição do exame. As barras de erro representam o intervalo de confiança a 95\% (CV - cardiovascular). 
minino (distância interquartil: 44-74 anos). A média de requisições por médico foi de 96,7 (IC95\%: 50,7-142,6). No quadro I apresentam-se em resumo os dados demográficos básicos da amostra.

Quanto aos motivos para a requisição, 48,5\% (IC95\%: 45,2-51,8\%) dos ECGs foram justificados pela presença de sintomas na consulta, 27,6\% (IC95\%: 24,7-30,7\%) pelo seguimento de doença cardiovascular previamente diagnosticada e 23,9\% (IC95\%: 21,2-26,9\%) pelo contexto de um exame global de saúde.

Na análise dos diagnósticos encontrados, 54,5\% (IC95\%: 51,2-57,8\%) dos ECGs eram normais, 35,9 \% (IC95\%: 32,7$39,1 \%$ ) apresentavam anormalidades minor e 9,7 (IC95\%: 7,9-11,8\%) apresentavam anormalidades major.

Os processos dos utentes foram revistos no sentido de determinar a alteração clínica face ao que se encontrava previamente à realização do ECG seis meses após. Nos 870 exames estudados, 67,9\% (IC95\%: 64,8-70,0\%) mantiveram os cuidados já instituídos sem qualquer alteração, em 13,8\% (IC95\%:11,7-16,3\%) alterou-se a medicação cardiovascular, em 12,3 \% (IC95\%: 10,3-14,7\%) foram requisitados novos exames auxiliares de diagnóstico, e 5,7\% (IC95\%: $4,8-7,5 \%)$ foram referenciados a uma consulta hospitalar. Registaram-se 2 óbitos neste período.

Quando se analisa a alteração à orientação em função dos motivos para a requisição dos ECGs (figuras 1 e 2), verifica-se que em todos os grupos a maior proporção corresponde à manutenção dos cuidados previamente instituídos com 60,2\% (IC95\%: 54,5-66,1\%) quando a sintomatologia na consulta era o motivo para o pedido do exame, 63,3\% (IC95\%: 55,4-71,3\%) nos exames de seguimento do doença previamente diagnosticada e 88,9\% (IC95\%: 79,8$98,1 \%$ ) quando o exame é requisitado no contexto do exame global de saúde $(p<0,001)$. O mesmo se verifica quan-

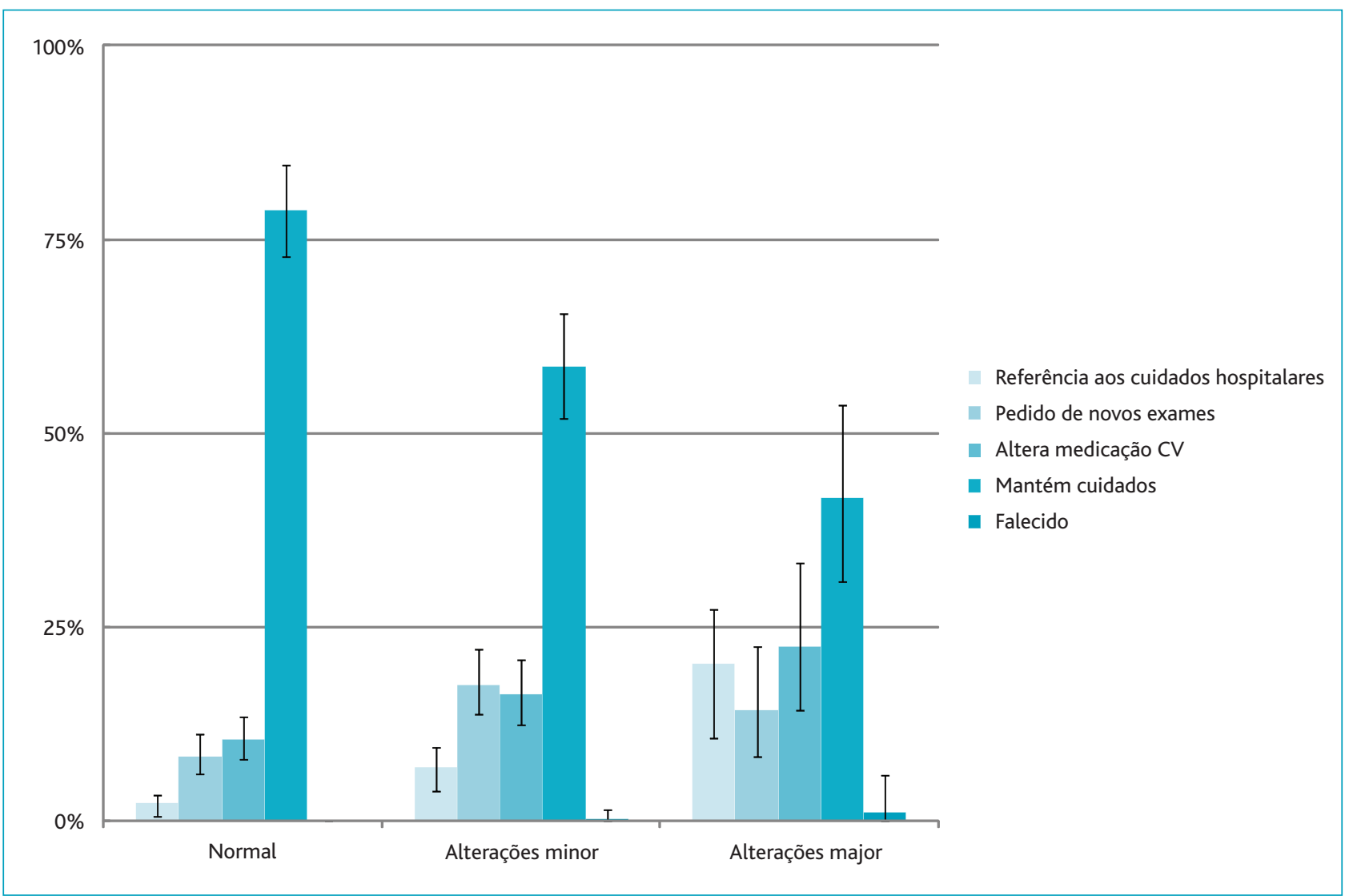

Figura 2. Estudo do seguimento dos utentes em função do resultado do exame. As barras de erro representam o intervalo de confiança a 95\% (CV - cardiovascular). 

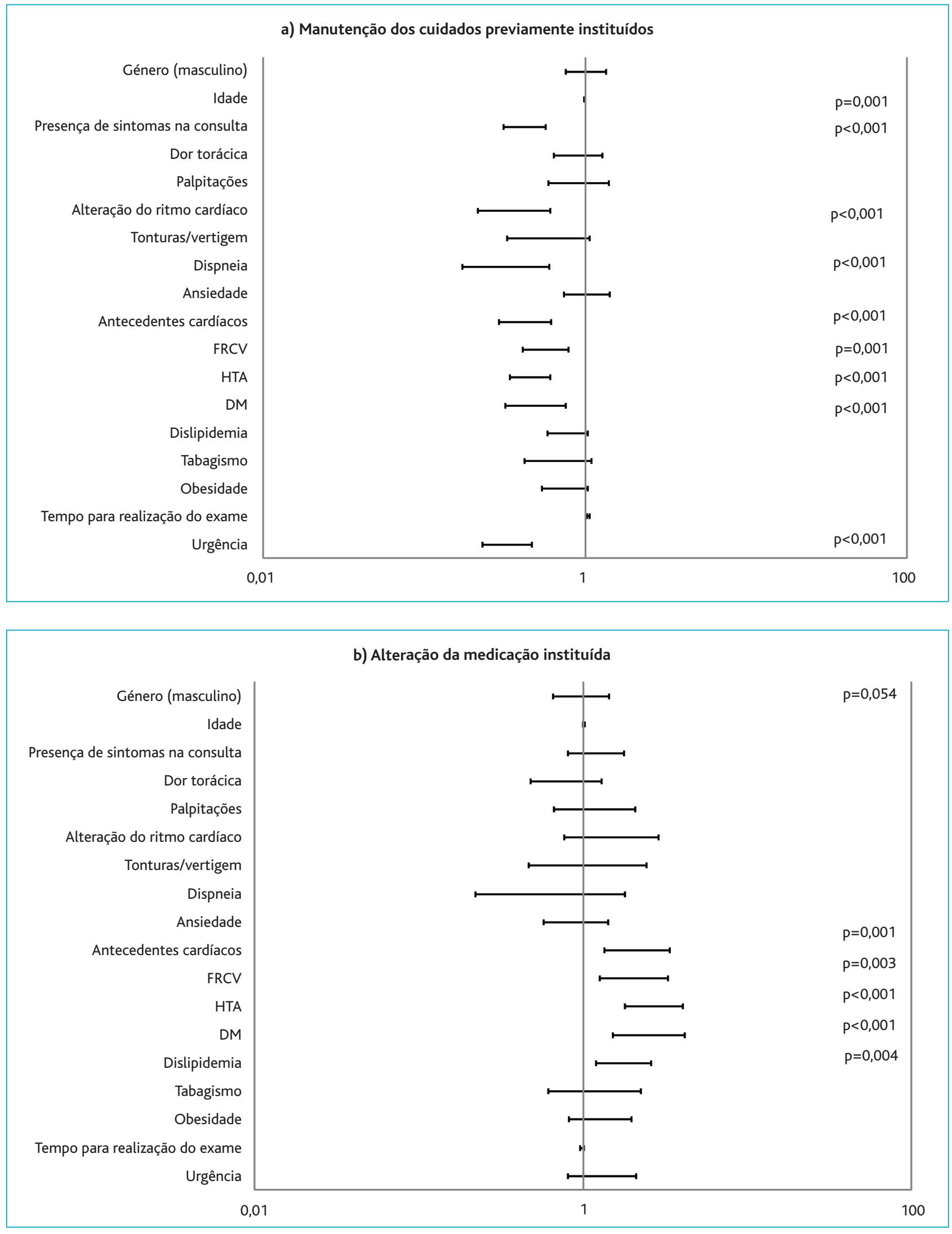

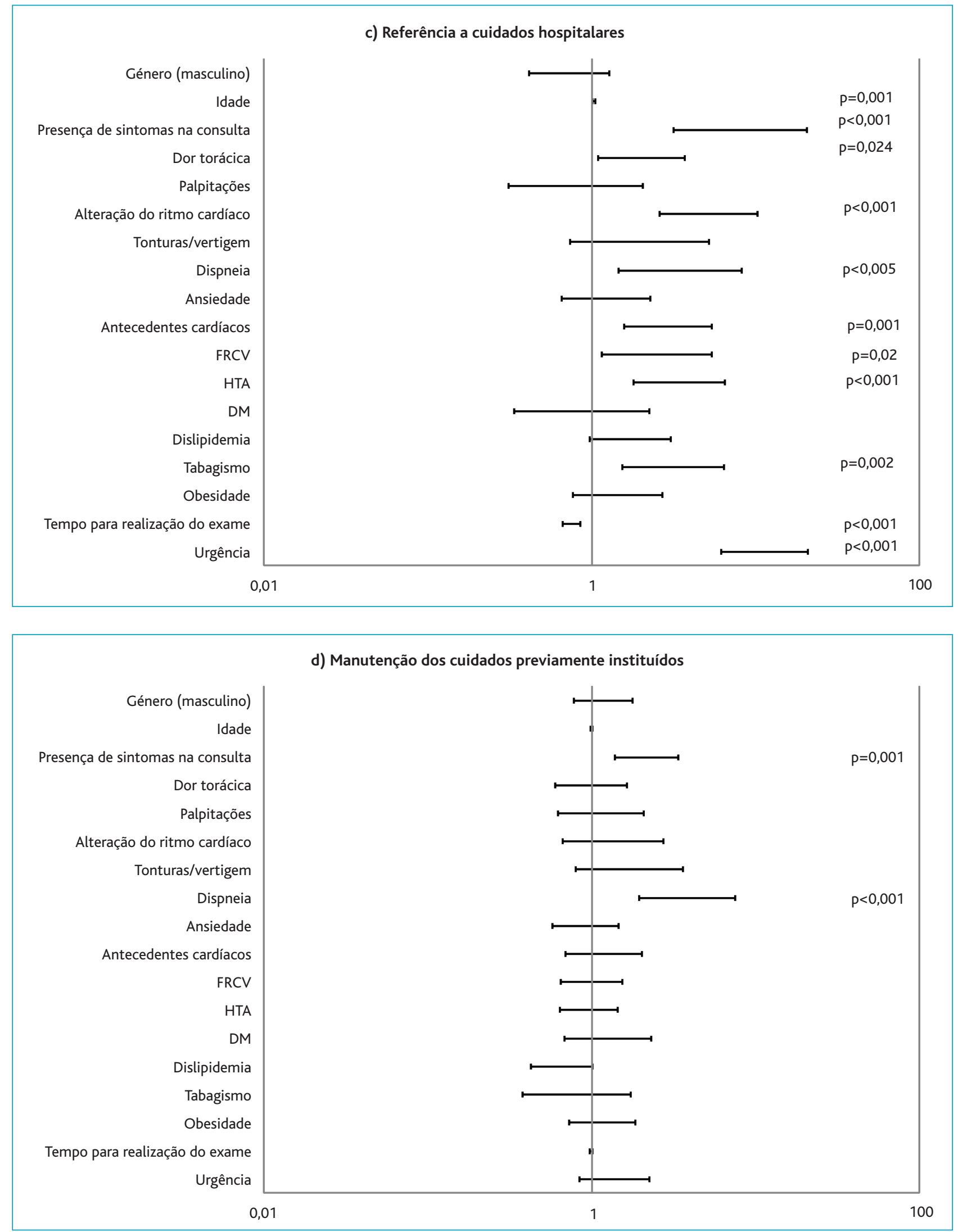

Figura 3. Análise univariada das condicionantes para a alteração ao seguimento previamente definido para o utente. As barras representam o intervalo de confiança a $95 \%$ dos valores de odds ratio. Na coluna à direita são mostrados os valores de $p$ nas variáveis com significância estatística. a) manutenção de cuidados previamente instituídos; b) alteração da medicação cardiovascular instituída; c) referência aos cuidados hospitalares; d) pedido de outros exames complementares. 
QUADRO II. Alteração ao seguimento dos utentes que realizaram ECG em função do motivo para o pedido e do resultado do exame (modelo de regressão logística multinominal).

A manutenção de cuidados previamente instituídos serviu de comparador em relação às restantes categorias da variável de resultado. São apresentados os valores de odds ratio (OR), os respetivos intervalos de confiança a 95\% (IC95\%) e o valor de $p$, calculado pelo teste da razão de verosimilhança para o modelo multivariado.

\begin{tabular}{|c|c|c|c|c|c|}
\hline Variável & $\begin{array}{l}\text { Manutenção } \\
\text { de cuidados }\end{array}$ & $\begin{array}{l}\text { Alteração da } \\
\text { medicação } \\
\text { cardiovascular }\end{array}$ & $\begin{array}{c}\text { Pedido de outros } \\
\text { exames } \\
\text { complementares }\end{array}$ & $\begin{array}{l}\text { Referência aos } \\
\text { cuidados } \\
\text { hospitalares }\end{array}$ & \\
\hline & OR (IC95\%) & OR (IC95\%) & OR (IC95\%) & OR (IC95\%) & $p$ \\
\hline Motivo para a requisição do ECG & & & & & $<0,001$ \\
\hline Exame global de saúde & 1,00 & 1,00 & 1,00 & 1,00 & \\
\hline Sintomatologia na consulta & 1,00 & $1,75(0,64-4,79)$ & $1,38(0,51-3,70)$ & $3,82(0,36-40,84)$ & \\
\hline $\begin{array}{l}\text { Seguimento de doença } \\
\text { previamente diagnosticada }\end{array}$ & 1,00 & $4,66(2,10-10,32)$ & $2,75(1,26-6,00)$ & $5,59(0,61-51,06)$ & \\
\hline Resultado do ECG & & & & & $<0,001$ \\
\hline Normal & 1,00 & 1,00 & 1,00 & 1,00 & \\
\hline Alteração minor & 1,00 & $1,89(1,17-3,06)$ & $3,31(2,00-5,48)$ & $3,72(1,60-8,91)$ & \\
\hline Alteração major & 1,00 & $2,70(1,28-5,70)$ & $3,09(1,32-7,28)$ & $10,11(3,54-28,88)$ & \\
\hline
\end{tabular}

do se procede à análise em função dos resultados dos ECG com 78,7\% (IC95\%: 72,8-84,6\%) para os exames normais, 58,7\% (IC95\%: 51,9-65,4\%) para as alterações minor e 41,7\% (IC95\%: 31,0-53,6\%) para as alterações major $(\mathrm{p}<0,001)$.

Estudaram-se as variáveis associadas à alteração ao seguimento dos utentes em análise univariada (figura 3). A manutenção dos cuidados previamente instituídos esteve inversamente associada à presença de sintomas na consulta (OR=0,416; IC95\% 0,308-0,563), sobretudo as alterações do ritmo cardíaco e a dispneia, aos antecedentes de doença cardiovascular (OR=0,421; IC95\%:0,290-0,610), à presença de fatores de risco cardiovasculares $(\mathrm{OR}=0,565$; IC95\%:0,407-0,783) e ao pedido de urgência na realização do ECG (OR=0,327; IC95\%: 0,230-0,466). A alteração da medicação cardiovascular foi mais frequente nos utentes com antecedentes cardiovasculares (OR=2,130; IC95\%: 1,347$3,336)$ e com fatores de risco cardiovasculares $(\mathrm{OR}=2,046$; IC95\%: 1,267-3,305), nomeadamente hipertensão arterial, diabetes mellitus e dislipidemia. O pedido de novos exames esteve associado positivamente aos utentes com sintomas na consulta (OR=2,156; IC95\%: 1,387-3,356), sobretudo nos que se apresentavam com dispneia. A referência aos cuidados hospitalares foi mais comum nos doentes mais idosos (OR=1,029; IC95\%: 1,012-1,047), nos que apresentaram sintomatologia na consulta $(\mathrm{OR}=8,082$; IC95\%: 3,179-20,546), como dor torácica, dispneia e alterações do ritmo cardíaco, nos que referiram antecedentes de doença cardiovascular (OR=2,929; IC95\%: 1,585-5,410), nos utentes com fatores de risco cardiovasculares $(\mathrm{OR}=2,500$; IC95\%: 1,159-5,393), como hipertensão arterial e tabagismo, e nos utentes a quem o exame foi requisitado com urgência (OR=11,317; IC95\%: 6,131-20,890) e foi menos comum em função do tempo que decorreu até à realização do exame desde o momento da sua requisição (OR=0,756; IC95\%:0,669-0,854). O médico que procedeu à requisição do exame foi também uma variável que demonstrou significância estatística na análise univariada $(\mathrm{p}<0,001)$, havendo profissionais que procederam ao pedido de novos exames e à alteração da medicação cardiovascular de forma significativamente diferente dos restantes.

O modelo de regressão logística multinominal foi utilizado para estudar a relação entre a alteração ao seguimento dos utentes que realizaram um ECG no contexto da consulta de MGF e as variáveis motivo para a requisição do exame e o seu resultado segundo a classificação de Novacode. O quadro II mostra os resultados após ajustamento para gé- 
nero, idade, médico que procedeu à requisição, presença de sintomas na consulta, antecedentes pessoais de doença cardiovascular, presença de fatores de risco cardiovasculares e o pedido do exame com urgência. Os resultados mostram que os ECG requisitados pela presença de sintomatologia na consulta não diferiram de forma significativa dos que o foram por rotina, ao passo que, no seguimento de doença previamente diagnosticada, o ECG foi um fator significativo de decisão clínica. Por outro lado, a decisão é significativamente alterada em função do ECG apresentar alterações minor ou major versus normal.

\section{DISCUSSÃO}

Este estudo diz-nos que cerca de dois terços dos ECGs requisitados na consulta de Medicina Geral e Familiar não interferem na abordagem clínica do utente que já se encontrava definida no momento em que se procedeu ao pedido do exame. Este dado é particularmente evidente nos ECGs requisitados no contexto de sintomatologia que o doente apresentou na consulta de Medicina Geral e Familiar, independentemente da alteração encontrada no exame. Por outro lado, nos doentes para seguimento de doença previamente diagnosticada, o ECG mostrou ser um elemento importante na definição da orientação clínica dos doentes. Da mesma forma, o facto de se verificar uma anormalidade minor ou major na leitura do ECG foi um importante fator de decisão clínica, independentemente do motivo que levou à sua requisição.

Em estudos anteriores, Rutten (2000) encontrou uma proporção de alterações à abordagem previamente definida de $31 \%{ }^{11}$ e van Dijke, ${ }^{15}$ em 1998, de $40 \%$. Estudos mais antigos mostraram variações menores de 7 e $16 \% .{ }^{16,17}$ Neste estudo o valor encontrado foi de $32,1 \%$, o que se situa na linha de atuação dos Médicos de Família.

Uma limitação deste estudo é o facto de não se ter considerado na análise a possibilidade de o resultado do ECG poder alterar no imediato a decisão do médico no momento em que este é realizado. Um dado indireto mas com relevância nesta discussão é o pedido de urgência na realização do ECG que se encontra significativamente associado a uma maior referência aos cuidados hospitalares, com um odds ratio de 11,317 e a uma menor manutenção de cuidados previamente instituídos ( $\mathrm{OR}=0,327$ ), o que pode significar que, no contexto de doença aguda, o ECG se assume como um importante elemento de decisão clínica. A proporção de alteração do seguimento nestes doentes foi de
53,5\% (IC95\%: 45,7-61,1\%), o que leva a pensar na especificidade da valorização das queixas no contexto da Medicina Geral e Familiar, sendo possível que um episódio de dor torácica seja abordado como potencialmente de causa cardíaca, mesmo que a história clínica aponte num outro sentido, dependendo da interação médico-doente característica desta especialidade. Um estudo desenhado para responder a esta questão poderia passar pela seleção de doentes com queixas agudas, verificando na variável de resultado o efeito da realização do eletrocardiograma.

O facto de se terem excluído os ECGs realizados fora das instalações do Centro de Saúde, representando uma proporção marginal, mas não passível de quantificação, da totalidade dos exames requisitados, é um viés a considerar na análise dos resultados, não parecendo no entanto suficiente para colocar em causa as conclusões.

Quando olhamos para os profissionais de saúde, é interessante verificar diferenças significativas em relação à orientação dada aos utentes em função dos motivos para o pedido de ECG e da sua interpretação. Na análise multivariada, os médicos procederam ao pedido de novos exames e à alteração da medicação cardiovascular de forma diferenciada uns dos outros, mas não na referenciação aos cuidados hospitalares. Este dado levanta a questão da variabilidade dos médicos no seu exercício diário, já descrita por Stafford, ${ }^{10}$ com parâmetros de atuação suficientemente diferentes a merecer estudo complementar específico.

Mais do que respostas definitivas, este estudo deixa questões ao nível da variabilidade do exercício médico em Medicina Geral e Familiar, ao nível da interação médico-doente, e ao nível da forma como os utentes reagem ao pedido e realização dos exames complementares de diagnóstico. Outros trabalhos com objetivos mais concretos poderão encontrar as respostas adequadas.

\section{CONCLUSÃO}

A maioria dos utentes que realizam um ECG na consulta de Medicina Geral e Familiar mantêm inalterados os cuidados instituídos previamente à requisição do exame. A sintomatologia cardíaca na consulta é o principal motivo de requisição do ECG mas não está associada a alterações ao seguimento nestes doentes. A utilidade do ECG verifica-se sobretudo nos casos de seguimento de fatores de risco cardiovasculares e de doença cardíaca prévia.

Artigo escrito ao abrigo do novo acordo ortográfico. 


\section{AGRADECIMENTOS}

Aos médicos, enfermeiros e secretários clínicos do Centro de Saúde de S. João, Porto, pelo trabalho quotidiano dedicado e pela forma como colaboraram e se interessaram nos trabalhos deste estudo.

\section{REFERÊNCIAS}

1. Einthoven W. Galvanometrische registratie van het menschilijk electrocardiogram. Disponível em: http://www.ecglibrary.com [acedido em 24/04/2010].

2. Davies A. Electrocardiographs in general practice. BMJ 1989 Aug 12; 299 (6696): 408-9.

3. Patel $P M, W u W C$. The electrocardiogram in the primary care office. Prim Care 2005 Dec; 32 (4): 901-30, vi.

4. Wellens HJ, Gorgels AP. The electrocardiogram 102 years after Einthoven. Circulation 2004 Feb 10; 109 (5): 562-4.

5. Macallan DC, Bell JA, Braddick M, Endersby K, Rizzo-Naudi J. The electrocardiogram in general practice: its use and its interpretation. J R Soc Med 1990 Sep; 83 (9): 559-62.

6. Conry CM, PaceWD, Main DS. Practice style differences between family physicians and internists. J Am Board Fam Pract.1991 Nov-Dec; 4 (6): 399-406.

7. Direção-Geral da Saúde. Elementos Estatísticos: Informação Geral: Saúde 2006. Lisboa: Direcção Geral da Saúde; 2008.

8. Direção-Geral da Saúde. Elementos Estatísticos: Saúde/97. Lisboa: DireçãoGeral da Saúde; 1999.

9. Froom J, Froom P. Electrocardiogram abnormalities in primary care patients. J Fam Pract 1984 Feb; 18 (2): 223-5.

10. Stafford RS, Misra B. Variation in routine electrocardiogram use in academic primary care practice. Arch Intern Med 2001 Oct 22; 161 (19): 2351-5.
11. Rutten FH, Kessels AG, Willems FF, Hoes AW. Electrocardiography in primary care; is it useful? Int J Cardiol 2000 Jul 31; 74 (2-3): 199-205.

12. Milhorn HT Jr, Robbins JG, Randolph R. Electrocardiograms in office practice. Fam Pract Res J 1986 Summer; 5 (4): 226-30.

13. Hespanhol A, Malheiro A, Pinto AS. O Projecto «Tubo de Ensaio» - breve história do Centro de Saúde S. João. Rev Port Clin Geral 2002 Mai-Jun;18 (3): 171-86.

14. Rautaharju PM, Park LP, Chaitman BR, Rautaharju F, Zhang ZM. The Novacode criteria for classification of ECG abnormalities and their clinically significant progression and regression. J Electrocardiol 1998 Jul; 31 (3): 157-87.

15. van Dijke MJ, van den Berg WN, Hoes AW. Beleidsverandering op grond van een ECG bij patienten in de huisartspraktijk. Ned Tijdschr Geneeskd 1998 Jun 13; 142 (24): 1383-7.

16. Fyfe T, Maclean NM.A health centre E.C.G. services: its use and abuse. Br Med J 1975 Mar 8; 1 (5957): 563-6.

17. Jones R. Electrocardiographic diagnosis in general practice. Practitioner 1984 Jan; 228 (1387): 85-7.

\section{ENDEREÇO PARA CORRESPONDÊNCIA}

Paulo Santos

Departamento de Clínica Geral

Faculdade de Medicina da Universidade do Porto

Alameda Prof Hernâni Monteiro

4200-319 PORTO, Portugal

e-mail:psantosdr@med.up.pt

Recebido em 28/12/2011

Aceite para publicação em 04/08/2012

\section{ABSTRACT}

\section{FOLLOW-UP OF PATIENTS AFTER ROUTINE ELECTROCARDIOGRAM TESTING IN PRIMARY CARE}

Background: Electrocardiography (ECG) is commonly used in primary care to study and follow patients with cardiovascular diseases.

Aim: The purpose of this study was to assess changes in patient care after performance of an ECG during a primary care office visit.

Methods: A prospective observational study was conducted at S. João Health Center, Oporto of all patients for whom an ECG was requested at primary care office visits over a two-year period, from 1/03/2007. Each time an ECG was requested, doctors recorded the reason for the test, the patient's symptoms, and past medical history. Electrocardiograms were saved for later analysis. Clinical files were hand searched six months after the ECG to assess changes in patient care. A multinomial logistic regression model was used to test associations between results of the ECG, classified by Novacode, and the indications for the test. Results: We studied $870 \mathrm{ECG}$, from 817 patients ( $56,4 \%$ females) with a median age of 57 years. The main reason for requesting an ECG was the presence of symptoms (48,5\%). Analysis of the results of the ECG showed that $54,5 \%$ were interpreted as normal, $35,9 \%$ had minor abnormalities, and 9,7\% showed major abnormalities. Care of the patient remained unchanged after $67,9 \%$ of tests (CI95\%: 64,8-70,0\%), and referral for hospital care occurred after 5,7\% (Cl95\%: 4,8-7,5\%). In multivariate analysis, the decision to change patient care was significantly influenced by ECG results in patients in whom the test was requested for follow-up of known cardiovascular disease but not for those who reported symptoms at office visit.

Conclusion: The ECG does not change the care of most patients who have the test done at an office visit. The greatest utility of the test was seen among patients with known cardiovascular risk factors or a past history of cardiovascular disease.

Key-words: Electrocardiography; Family Practice; General Practice; Disease Management; Medical History Taking. 